\title{
Analysis and Treatment of Infection Factors of Incision in Acute Appendicitis
}

\author{
Ruizhi Hou, Yan Liu \\ China-Japan Union Hospital of Jilin University, Changchun, Jilin, 130021, China
}

Keywords: Acute appendicitis, Operative incision infection, Related factors, Preventive measures

\begin{abstract}
In order to analyze the related factors of incision infection in acute appendicitis, the paper put forward effective treatment measures. A total of 371 patients with acute appendicitis were selected from January, 2016 to December, 2017 in China-Japan Union hospital. Moreover, the statistical analysis included different genders, ages and incisions, operation time, abdominal incision irrigation, drainage, pathological types, perforation and complications of the incidence of incision infection. The authors analyzed related factors of acute appendicitis surgical incision infection, and put forward effective treatment measures. According to the analysis of the related factors of incision infection in acute appendicitis, the results showed that the infection of acute appendicitis operation was related to the course of disease, the type of incision, the time of operation, the irrigation of abdominal incision and the situation of drainage. Perforation and complications are relevant. In conclusion, if the patients in acute appendicitis appeared the longer course of disease, exploratory incision, longer operation time, massive abdominal incision irrigation, the situation of drainage, perforation, complications, the incidence of incisional infection would be increased. Therefore, preventive measures must be taken to improve the curative effect of the operation and promote the patient's health.
\end{abstract}

\section{Introduction}

Appendicitis is one of the most common surgical diseases in hospital. It is mainly treated by excision and is the most basic clinical treatment. However, appendicitis is in acute appendicitis surgery. The most common complication of surgical incision infection is high, so we must do a good job in the prevention and treatment of acute appendicitis incision infection, so that to improve the efficacy. The purpose of this study was to analyze the factors and effective management of incision infection in acute appendicitis.

\section{Data and Methods}

\subsection{Clinical Data}

The clinical data of 371 appendicitis patients from January to December 2017 in our hospital were studied. 181 cases were male and 190 cases were female. And the age is 14 to 75 years, and the average age was $47.2 \pm 6.8$ years.

Symptoms of patients were fever, nausea, vomiting, abdominal pain right discomfort, abdominal pain and so on, which excluded patients with serious cardiovascular disease, immune diseases, tumor diseases, blood diseases. Therefore, it can be ensured that the results would not be affected.

\subsection{Methods}

Pre-operative preparation: The auxiliary examination must be perfected as soon as possible. With the exception of a few patients whose whole body condition is extremely poor, or whose diagnosis is not clear, emergency surgery should be performed as soon as possible, and antibiotics should not be routinely used before operation. Incision selection and protection: In the cases with definite diagnosis, the right lower abdominal incision or the McDonnell transverse incision were routinely selected. For patients who need abdominal cavity exploration or patients with diffuse peritonitis signs during surgery with unclear preoperative diagnosis, the right middle and lower abdomen incision via rectus abdominis or paracentric exploration is selected. The common scalpel is used to 
cut the skin and subcutis, and the incisions are cut sharply to separate each layer of the abdominal wall. During the operation, the appendices are separated by gauze, and the infected appendicitis are not touched by the hands as far as possible, so as to avoid the wound contamination.

Drainage: In principle there is no need to place drainage. In addition, few gangrene perforated appendicitis with diffuse peritonitis and abdominal cavity with a large amount of viscous pus patients need drainage. In diffuse peritonitis, the pus viscous can be washed. Moreover, they should be washed with proper amount of saline multiple times.

Post-operative treatment: The patients were routinely treated with antibiotics and anti-infection after operation. The third generation of cephalosporin, ornidazole and antiinfective therapy are generally given to those with pyosis, gangrene and perforation. After the operation, the patient should be replaced with incision dressing every other day. If there is inflammatory reaction in the incision, or if the wound is changed with ethanol or iodophor wet compress many times, magnesium sulfate can also be used to promote the healing of the wound.

\subsection{Statistical Processing}

The data were processed by SPSS 19.0 software, and the count data were analyzed by $\chi^{2}$ test $(\mathrm{P}<$ 0. 05).

\section{Results}

According to the analysis of the related factors of incision infection in acute appendicitis, the results showed that the infection of acute appendicitis operation was related to the course of disease, the type of incision, the time of operation, the irrigation of abdominal incision and the situation of drainage, perforation and complications, etc. (See in Table 1)

Table 1. Analysis of Factors Related to Incision Infection in Acute Appendicitis.

\begin{tabular}{|c|c|c|c|c|}
\hline \multicolumn{2}{|r|}{ Clinical Data } & Infection of Incisional & $\chi^{2}$ & $\mathrm{P}$ \\
\hline \multirow{2}{*}{ Gender } & Male(n=194) & $8(4.1)$ & \multirow{2}{*}{0.482} & \multirow{2}{*}{$>0.05$} \\
\hline & Female(n=177) & $4(2.3)$ & & \\
\hline \multirow{2}{*}{ Age } & $\leq 60$ Years Old $(n=309)$ & $8(2.6)$ & \multirow{2}{*}{0.589} & \multirow{2}{*}{$>0.05$} \\
\hline & $>60$ 岁 $(n=62)$ & $4(6.5)$ & & \\
\hline \multirow{2}{*}{ Course of Disesse } & $\leq 24 h(n=320)$ & $6(1.9)$ & \multirow{2}{*}{4.892} & \multirow{2}{*}{$<0.05$} \\
\hline & $>24 h(n=510)$ & $6(11.8)$ & & \\
\hline \multirow{2}{*}{ Incision Type } & McIntosh Incision $(\mathrm{n}=341)$ & $5(1.5)$ & \multirow{2}{*}{5.481} & \multirow{2}{*}{$<0.05$} \\
\hline & Exploratory Incision $(n=30)$ & $7(23.3)$ & & \\
\hline \multirow{2}{*}{ Operation Time } & $\leq 1 \mathrm{~h}(\mathrm{n}=306)$ & $5(1.5)$ & \multirow{2}{*}{6.549} & \multirow{2}{*}{$<0.05$} \\
\hline & $>1 h(n=65)$ & $7(10.8)$ & & \\
\hline \multirow{2}{*}{$\begin{array}{c}\text { Abdominal } \\
\text { Incision Irrigation }\end{array}$} & A Large Number $\quad(n=25)$ & $10(40.0)$ & \multirow{2}{*}{6.893} & \multirow{2}{*}{$<0.05$} \\
\hline & Partial or Unwashed $(n=346)$ & $2(0.6)$ & & \\
\hline \multirow{2}{*}{ Drainage Situation } & Not Have $(n=334)$ & $4(1.2)$ & \multirow{2}{*}{5.486} & \multirow{2}{*}{$<0.05$} \\
\hline & Have $(n=37)$ & $8(21.6)$ & & \\
\hline \multirow{4}{*}{ Pathological Types } & Purity $(n=84)$ & $1(1.2)$ & \multirow{4}{*}{0.475} & \multirow{4}{*}{$>0.05$} \\
\hline & Suppurative $(n=152)$ & $4(2.6)$ & & \\
\hline & Honeycomb Inflammation $(n=104)$ & $4(3.8)$ & & \\
\hline & Gangrenous $(n=31)$ & $3(9.7)$ & & \\
\hline \multirow{2}{*}{$\begin{array}{c}\text { Perforation } \\
\text { Condition }\end{array}$} & Perforated $(\mathrm{n}=25)$ & $6(24.0)$ & \multirow{2}{*}{4.898} & \multirow{2}{*}{$<0.05$} \\
\hline & No perforation $(n=346)$ & $6(1.7)$ & & \\
\hline \multirow{2}{*}{ Complications } & Have Complications $(\mathrm{n}=20)$ & $5(25.0)$ & \multirow{2}{*}{5.036} & \multirow{2}{*}{$<0.05$} \\
\hline & No complications(n=351) & $7(2.0)$ & & \\
\hline
\end{tabular}




\section{Discussions}

Appendicitis is a common surgical disease. Most of the patients were treated with pain in the right lower abdomen. The clinical manifestations were metastatic right lower abdominal pain accompanied by fever nausea and vomiting. The blood routine examination showed that the white blood cell count and neutrophils were significantly increased. The main signs of the patients were tenderness in the periumbilical area. At present, the main clinical treatment for acute appendicitis is resection and surgery, which is also the most basic clinical treatment. The most common complication is surgical incision infection, which is a serious threat to postoperative recovery, and also an important factor threatening the life of patients. Therefore, it is necessary to strengthen the prevention and treatment of surgical incision infection and improve the effect of surgical treatment.

In order to study the influencing factors of incision infection in acute appendicitis surgery, some of the acute appendicitis patients were selected as the research objects. The incidence of incision infection, including gender, age, incision type, operative time, abdominal incision irrigation, drainage, pathological type, perforation and complications, was analyzed statistically. Statistics show that the infection of incision in acute appendicitis is related to course of disease, type of incision, duration of operation, irrigation of abdominal incision, drainage, perforation and complications, etc. The longer the course of acute appendicitis, the longer the exploratory incision, the longer operation time, a large number of abdominal incision irrigation, drainage, perforation and complications will increase the incidence of incision infection. Therefore, we need to do a good job of preventive measures.

For elderly patients, incision infection is easy to occur because of the autoimmune mechanism and disease and other related factors. Therefore, in order to avoid incision infection in elderly patients, it is necessary to deal with basic diseases to ensure the health of elderly patients for obese patients. After the closure of the peritoneum, the incision should be cleaned in time to reduce postoperative infection and use iodophor solution to wash the incision, which could reduce the infection of incision. At the same time, it can also shorten the hospitalization time of the patients, so that to reduce the impact of the operation on the patients and improving the recovery rate of the patients. So it is one of the important ways to prevent incision infection during acute appendicitis.

If the operation time is too long, the bacteria around the incision and the bacteria in the air will be removed from the incision with the sweat of the human body, and the infection will occur. The long operation time can lead to the increase of stretch time, damage human local tissue, aggravate ischemia and hypoxia, reduce the antibacterial ability of local tissue and increase the incidence of infection. Therefore, the incision site should be selected in the process of operation to ensure that the incision site is close to the focus and easy for the operator to operate. At the same time, make sure that the incision is not too small, such as the incision is relatively small, and easy to lead to excessive, which will affect the incision tissue blood flow.

For acute appendicitis surgery, drainage tubes are generally not recommended. However, in patients with complicated appendicitis complicated with peritonitis, drainage tubes such as persistent but small amount of purulent exudation are required. The patient should be subjected to intraperitoneal drainage. However, the incidence of this phenomenon is relatively low in practice, and the common situation is intraoperative intraperitoneal empyema caused incision site infection. Therefore, in order to avoid this kind of infection, the drainage tube can be placed in the incision, and the proper puncture is carried out during the placement, so as to ensure the accurate position of the drainage tube. On the basis of unobstructed drainage, the incision infection caused by improper placement should be reduced.

\section{Suggestions}

In order to reduce the incidence of incision infection in acute appendicitis, first of all, vocational training should be done, and surgical personnel should strengthen their understanding of appendicitis operation. Appendicitis should not be regarded as a minor operation because it is an appendectomy, and we should be strictly aseptic to ensure the standardization of the operation. At 
the same time, we should guide and supervise the doctor in the process of teaching to prevent the wound infection caused by negligence and affect the patient's recovery after operation. We should manage the hospitalization of appendicitis patients, observe the postoperative incision and state of disease in time, and use antibiotics according to the relevant requirements. Secondly, we need to make early detection, early diagnosis and early treatment. When the patient has typical appendicitis symptoms, he should go to the hospital to see a doctor in time. After determining the symptoms, he should take treatment methods such as surgery to reduce postoperative infection. This can reduce the incidence of gangrene and purulent appendicitis, and help reduce the pain of patients. Further, for the elderly patients, appropriate measures should be taken in time to treat the underlying diseases, improve the patient's own resistance and reduce the incidence of surgical incision infection. For patients with peritonitis and obesity, abdominal irrigation, proper drainage and adequate hemostasis should be taken. For the patients with relatively thick fat layer, the use of electric knife was reduced, and the inactivated adipose tissue was washed and cleaned before suture, so as to reduce the incidence of incision infection. Therefore, in order to effectively reduce the incidence of incision infection, we must do a good job of preventive measures so that to improve the effectiveness of the operation and promote the recovery of patients.

\section{References}

[1] Yin Tao. Analysis and Treatment of Infection Factors of Incision in Acute Appendicitis [J]. Chinese Journal of Modern Medicine, 2013, 23(18): 68-70.

[2] Zhang Eryong, Zhang Chunjun, Huang Haifeng. Case-control Study on Related Factors of Incision Infection after Acute Appendicitis [J]. Chinese Journal of Nosocomial Infection, 2010, 20(05): 658-660.

[3] Lai Jianzhong. Analysis of Factors and Effective Management of Incision Infection in Acute Appendicitis [J]. Journal of Clinical Rational Drug Use, 2016, 9(30): 174-175.

[4] Yu Guojian. Analysis on Risk Factors of Incision Infection in Patients with Acute Appendicitis after Operation [J]. Contemporary Medicine Theory, 2014, 12(16): 213-214.

[5] Lu Xiaorong. A Retrospective Analysis of the Related Factors of Incision Infection after Acute Appendicitis [J]. Journal of Bai Qiuen Military Medical College, 2013, 11(06): 525-526.

[6] Zhou Sijun. Analysis and Countermeasure of Incision Infection after Acute Appendicitis [J]. Foreign Medicine (Medical Geography Division), 014, 35(04): 331-333. 